\title{
Perception of illuminance flow in the case of anisotropic rough surfaces
}

\author{
Jan J. Koenderink, Andrea J. van Doorn, and Sylvia C. Pont \\ Universiteit Utrecht, Utrecht, The Netherlands
}

\begin{abstract}
Human observers estimate the illumination direction of rough surfaces rather precisely. When surfaces are rough, the illumination generates visible "texture" from differential shading at the level of the roughness, whereas differential illumination at the level of significant global surface curvature leads to the more familiar "shading." The shading is used in conventional shape-from-shading (SFS) algorithms, which ignore the illumination texture cue. Because of this simplification, SFS algorithms are typically formulated as global problems (partial differential equations, etc.). Human observers are likely to apply different methods than do these conventional SFS algorithms, however. When the roughness is not isotropic, one expects systematic errors in the visual detection of illumination direction, conceivably giving rise to erroneous shape estimates. Here we addressed this issue through systematic psychophysics on illumination direction detection as a function of the roughness anisotropy. Our expectations were fully borne out, in that the observers committed the predicted systematic errors. These results are precise enough to allow the inference that illumination direction detection is based on second-order statistics - that is, of edge detector (rather than line detector) activity.
\end{abstract}

The shading cue in visual psychophysics and shape from shading (SFS) in machine vision have long histories (Horn \& Brooks, 1989; van Diggelen, 1959). The emphasis is usually given to shape inferences on the basis of an image of a smooth, illuminated surface, although a few authors have also considered the dual problem of light field, rather than shape, inferences (Pentland, 1982).

The shading that is found in the image results from perspective, surface properties, object geometry, and the light field (Gershun, 1939; Moon \& Spencer, 1981). The light field is technically known as the "radiance," a function that describes the luminous flux per unit area and unit solid angle at any point in space and in any direction. Usually only rather simplified cases are considered, such as orthographic projection (with foreshortening as the only perspective effect), smooth Lambertian surfaces (in which the viewing direction is irrelevant, only illumination direction is causally effective), and uniform, collimated beams. Moreover, the effects of vignetting (the object itself influencing the light field) and multiple scattering, or interreflection (the object itself acting as a secondary light source) are generally disregarded.

In most generic (i.e., daily-life) cases, these extreme simplifications do not apply at all. They do apply approximately in carefully controlled laboratory setups, though Lambertian surfaces can only be approximated, and vignetting and multiple scattering cannot be avoided. Thus, the "purest" cases of shading are encountered in computer graphics, where it is easy enough to adjust the laws of physics to one's liking. Many experiments in the psychophysics of shape are currently done in the con- text of such virtual scenes. Essentially all of the relevant theory (mostly in the context of machine vision) departs from these very restrictive and somewhat unrealistic initial assumptions.

In this (extreme) simplification, the shading is due solely to Lambert's attitude effect (Lambert, 1760); that is, the irradiance of surface patches is the cosine of the angle subtended by the light direction and the (inward) surface normal times the irradiance of a normally illuminated patch. Equivalently, the shading is proportional to the normal component of the "light vector"- that is (for a uniform, collimated beam), the direction of the light beam. If one knows the light vector, the SFS problem is well posed. If one does not (as in generic perception), both the shape and the light vector have to be determined, and one has to cope with a group of ambiguity transformations (Belhumeur, Kriegman, \& Yuille, 1999).

One aspect of real scenes that is ignored in this setting is the fact that actual surfaces are typically not smooth, but rough on a scale that is resolved in the image. One consequence is that the shading becomes ill defined at a point (or pixel) and has to be estimated as a local average, with the average taken over an area that includes significant variation due to the surface roughness. It is standard practice in machine vision to run SFS algorithms on slightly blurred images for this reason, and roughness on the microscale is then considered a nuisance. A more cheerful view is possible, though: One may consider the texture induced by the illuminated roughness to yield additional optical information (Penirschke, Chantler, \& Petrou, 2002; Pont \& Koenderink, 2003, 2004; Varma \&

J. J. Koenderink, j.j.koenderink@phys.uu.nl 
Zisserman, 2004). Completely textureless surfaces (i.e., surfaces that are smooth in terms of 3-D roughness) are rare in nature, but they do occur in the man-made environment. One would expect differences between shading cues in the cases of smooth and rough surfaces, a topic that deserves more attention than it conventionally receives.

Even a uniformly white Lambertian surface that is rough on the microscale (think of a globally "smooth" surface that has a roughness that is visually resolved) will show up as textured in the image (Koenderink \& Pont, 2002). The texture is obviously not due to surface markings or pigmentation (which is the type of texture implied in "shape from texture" discussions), but instead to shade and shadow at the scale of the surface undulations. In Figure 1 we show an example. The figure shows three flat (on the macroscale) surfaces that have microscale undulations whose depth profile is determined by a random sample of isotropic Gaussian noise. The noise has random amplitude with a Gaussian distribution (hence, "Gaussian noise") and has a rotationally symmetric autocorrelation function (hence, "isotropic"); the statistics are identical for all three of the samples. The difference is in the direction of illumination: If the direction is almost normal (Figure 1A), the shading looks highly ambiguous to the human observer. Many SFS algorithms fail in this case, though a few are especially designed for it (and for only this case). If the illumination direction is almost grazing the surface (Figure 1C), cast shadows dominate the view. Virtually all SFS algorithms fail in this case, too. If the illumination is "nice" (Figure 1B), we have the "shading regime," in which human perception generally does well, as do typical SFS algorithms. The texture in the image is not isotropic, even though the surface roughness is, because the direction of the illumination polarizes the texture. Formally, one has a texture that is generated by the tangential component of the light vector.

An object placed in a beam of light is revealed (in the sense that it gives rise to an image) in two major ways. The first is that the normal component of the direction of the beam generates a scalar field (a distribution of magnitude), conventionally denoted the "shading." The second is that the tangential component of the direction of the beam generates a vector field (or "flow") over the surface of the object, which might be called the "surface illuminance flow." The surface illuminance flow generates the illuminance texture. In the image, the surface illuminance flow appears as "image illuminance flow" that is revealed if texture due to surface roughness is present. An analysis of the image thus potentially yields inferences concerning both the normal and the tangential components of the light field, thus changing the conventional framing of the SFS problem decisively if the illuminance flow is detected. In the context of this article, the difference between surface illuminance flow and image illuminance flow is immaterial, because the viewing of the surface is always frontoparallel.

We have found that human observers are quite sensitive to the illuminance texture cue (Koenderink, van Doorn, \& Pont, 2004) and are able to estimate the direction of surface illuminance flow rather precisely, albeit with a $180^{\circ}$ ambiguity. It is very likely that the cue they use is contained in the set of spatial averages of products (including squares) of spatial mixed partial derivatives of image intensity, the average extending over many times the grain size of the texture. (See the Appendix.) Thus, the precise shading pattern is not resolved by the physiological mechanisms that extract illuminance flow (one may think of a [spatially] coarse measure of simple cell activity in the primary visual cortex). Because the direction of illuminance flow can be observed, the conventional SFS theories are in many circumstances irrelevant to the case of human visual perception, because it is unlikely that the observer will ignore informative cues the way these theories do.

Likely substrates for the ability to detect illuminance flow direction are the second-order statistics of either "edge detector" or "line detector" activity (or both) in the primary visual cortex. We have developed formal theo-
A

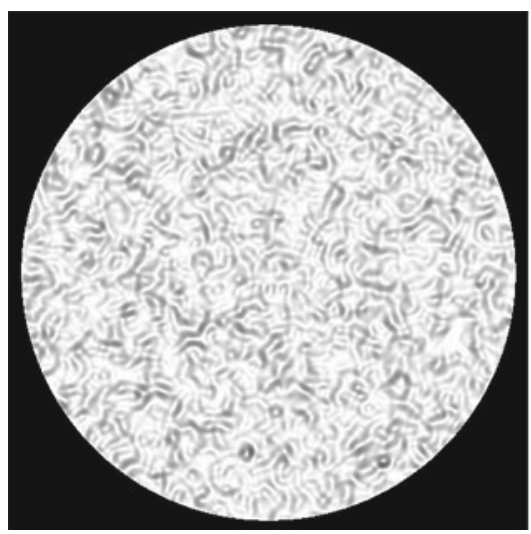

B

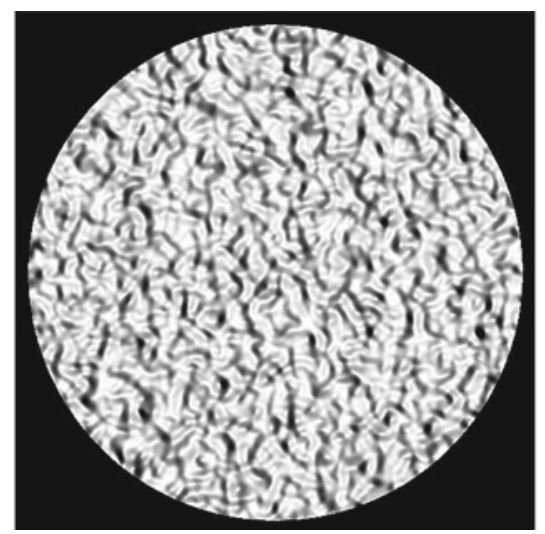

C

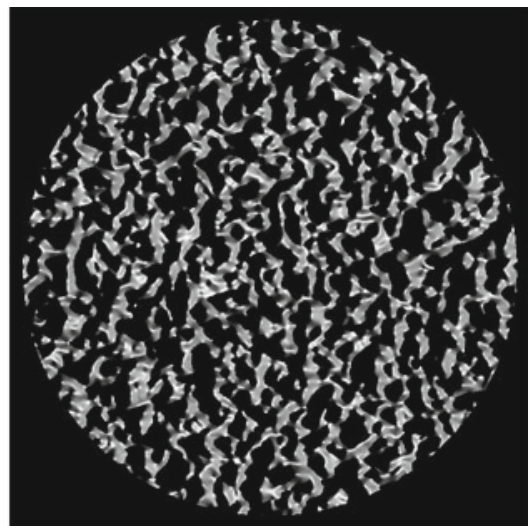

Figure 1. Three samples of illuminated isotropic surfaces with identical surface statistics. (A) An example of illumination in the second-order shading regime; $(B)$ an instance of the generic shading regime; $(C)$ an instance of the shadow-dominated regime. The psychophysical shading cue and the machine vision shape-from-shading algorithms apply only to the generic shading regime. 
A

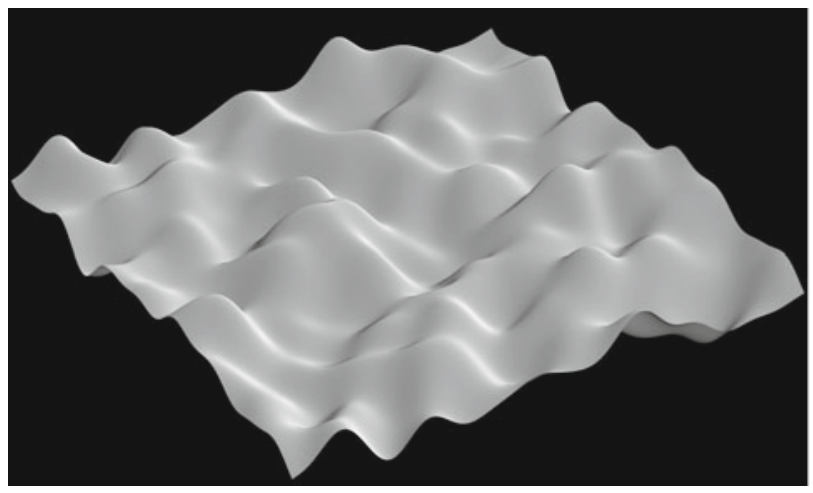

B

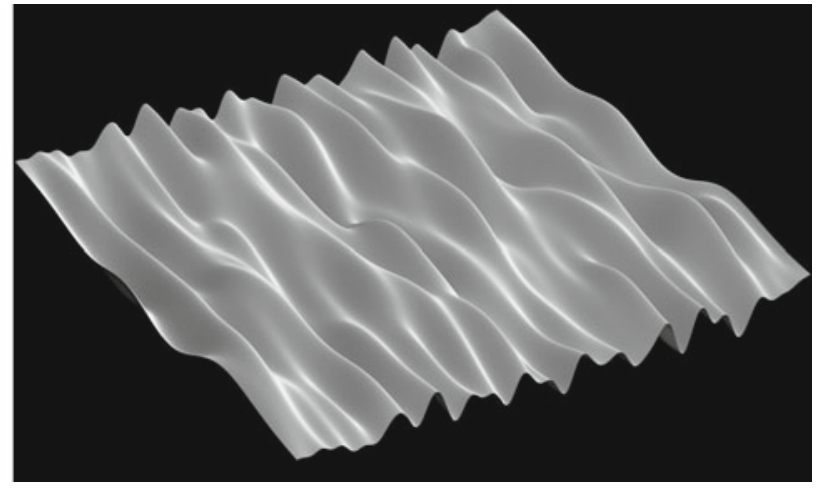

Figure 2. (A) An isotropic Gaussian rough surface. (B) A (very) anisotropic Gaussian rough surface.

ries that perform very robustly in machine vision implementations (Koenderink, van Doorn, Kappers, te Pas, \& Pont, 2003). It seems likely that the human visual system contains similar mechanisms. The way this works is as follows: The spatial power spectrum of the image of an illuminated rough surface is bimodal, with the orientation of the mode pair being along the direction of flow. This orientation can be obtained from the second-order statistics of either edge or line detectors. In computer vision, such detectors are known as "structure tensors" (Förstner, 1994; Harris \& Stevens, 1988; Knutsson, 1989; Köthe, 2003).

The formal theories assume isotropic surface roughness. Perhaps the majority of the rough surfaces encountered in the daily environment are of this type, but instances of anisotropic surface roughness are by no means rare (Räth \& Morfill, 1997). In Figure 2, we illustrate a sample of isotropic Gaussian roughness and a sample of (very) anisotropic Gaussian roughness. For such anisotropic surfaces, the simple isotropic theory does not work.
If you run "illuminance flow from texture" algorithms on images of illuminated anisotropic Gaussian noise, you will find systematic deviations from veridicality. We show an example of such a result in Figure 3. Thus, it may be expected that human observers will misperceive illumination direction in such cases, quite possibly leading to wrong shape inferences. Of course, this only applies to illumination texture. For anisotropic pigmentation texture (in which shape from texture rather than shape from shading is indicated), human observers perform well (Todd, Oomes, Koenderink, \& Kappers, 2002).

Figure 3 illustrates the same rough surface patch illuminated from three different directions. The normal component (and thus the global shading, which is evident from the average illuminance) was the same in these three cases; only the direction of the surface illuminance flow was varied. The anisotropy of the surface is very marked, like that of the surface illustrated in Figure 2B. The direction of elongation of the troughs and ridges of the surface in Figure 3 runs horizontally with respect to the page. In
A

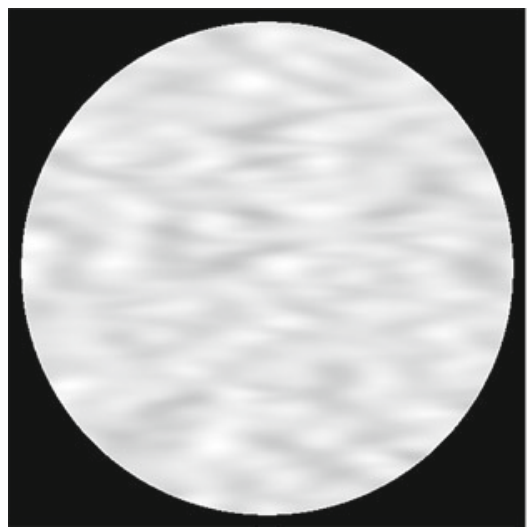

B

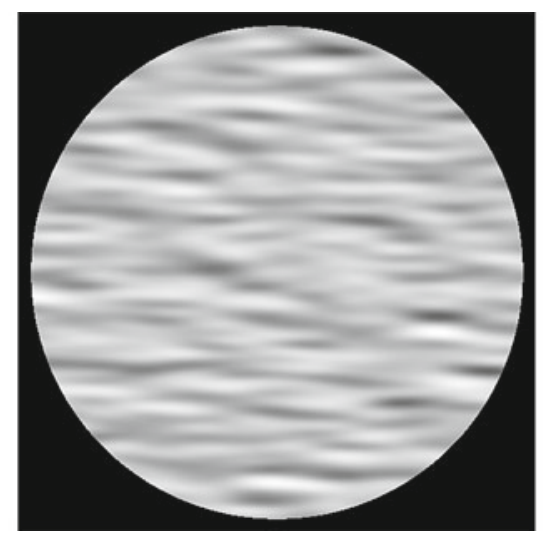

C

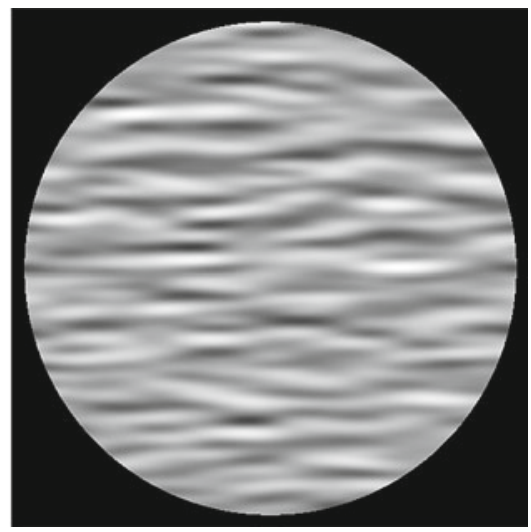

Figure 3. An example of a highly anisotropic surface, illuminated from three different directions. In the image at the left, the illuminance flow is along the extended troughs and ridges of the relief; in the image at the right, the illuminance flow is at a right angle to these undulations. For the center image, the illuminance flow is at $45^{\circ}$ from the direction of the elongated features. Notice the similarity between the center and right images, corresponding to common nonveridical human judgments, and notice that the image at left looks hardly like an illuminated surface at all. 
the image at left, the surface illuminance flow is along this direction, and in the image at right it is at right angles to it (vertically with respect to the page). Notice the enormous difference in appearance between the two images. For an isotropic surface, these images would have differed only by a $90^{\circ}$ rotation. The image at the left is hard to parse as an illuminated surface, because it effectively is in the regime illustrated in Figure 1A. (This is the case because significant changes of surface attitude occur only in a direction perpendicular to the direction of illumination.) In this regime, human observers also have problems with isotropic roughness. On the right side of Figure 3, we are fully in the "shading" regime, and the image looks like a rough surface illuminated from the actual (vertical) direction. The only ambiguity here is the $180^{\circ}$ ambiguity, since it is fully ambiguous whether the illuminance is from the top to the bottom of the page or from the bottom to the top.

The most interesting case is illustrated at the center of Figure 3. Here, the surface illuminance flow subtends an angle of $45^{\circ}$ to the direction of elongation of the surface undulations. Notice that this image does not look all that different from the image on the right, with the only difference being perhaps a lower contrast. The estimated direction of illuminance flow is again vertical, thus committing a $45^{\circ}$ systematic error! The angular coordinate system used in this article to indicate such directional errors is defined in Figure 4.

In this study, we considered the case of illuminated anisotropic rough surfaces and investigated the systematic errors in human surface illuminance flow estimates. Our

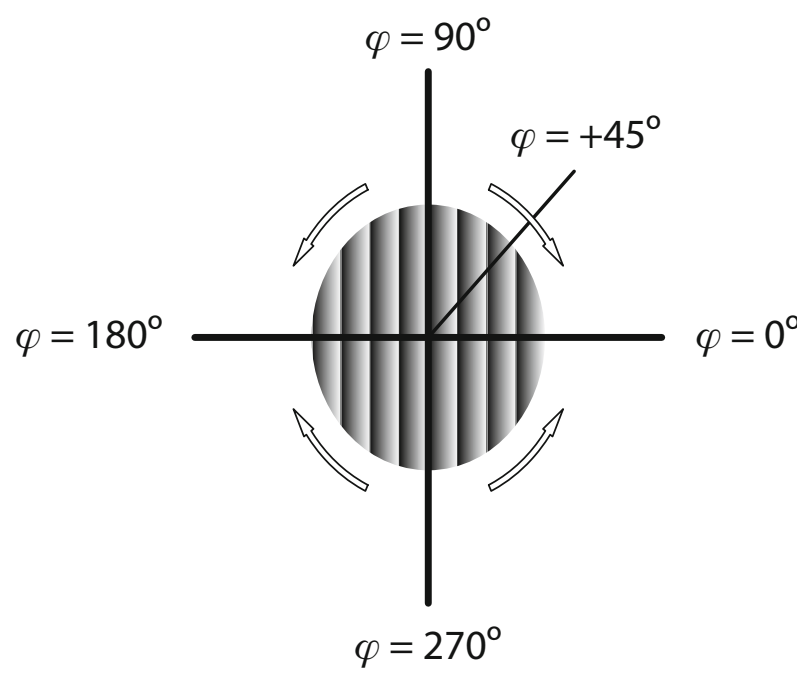

Figure 4. Definition of the angles. The axis of elongation is in the $90^{\circ}-270^{\circ}$ direction. The perceived direction of illumination shifts toward the direction perpendicular to the axis of elongation, as indicated by the arrows. Thus, a (veridical) illumination of $45^{\circ}$ will be perceived as closer to the $0^{\circ}$ direction, which we notate as a positive deviation. The deviations in the first quadrant are therefore positive, those in the second quadrant negative, those in the third quadrant positive, and those in the fourth quadrant negative. In the other figures, we plot only the fourth and first quadrants, since the direction of illumination is specified modulo $180^{\circ}$. initial hypothesis is that the human observer might simply apply the algorithm for surface flow estimation that works well for the isotropic case. If this is correct, one should be able to predict the systematic errors committed by observers when the illuminance texture is due to anisotropic rough surfaces.

\section{Theory}

If one illuminates a hill from one side and looks at it normally, one sees a "dipole" consisting of a light and a dark blob, with the direction from the center of the light to the center of the dark blob being the direction of the surface illuminance flow. Something similar happens for a local depression, with only the difference that the polarity of the dipole is reversed. A random Gaussian surface can be regarded as a random collection of hills and depressions, generating a collection of dipoles that all have the same orientation (direction modulo $180^{\circ}$ ) but random polarity. Thus, an average will show nothing, because the dipoles of different polarity cancel each other. In order to obtain the relevant statistics, one has to somehow "square" the dipoles (i.e., use second-order statistics), in which case the average reveals the overall dipole orientation (i.e., the illuminance flow direction, modulo $180^{\circ}$ ). When you look at power spectra of illuminated rough surfaces, you notice that such power spectra (which are second-order statistics) are bimodal with modes aligned along the direction of the surface illuminance flow.

Simple models use either the gradient or the Hessian of the image intensity. The "gradient" is a vector normal to the local edge, whereas the "Hessian" detects elongated blobs or lines (see Figure 5). Technically, one constructs the gradient-squared and Hessian-squared structure tensors, which is equivalent to looking at the second-order statistics of populations of edge detectors (in the case of the gradient) or line detectors (in the case of the Hessian). Notice that we use the terms "edge" and "line" detectors merely as conventional descriptive terms; it is now generally understood that these terms are misleading if interpreted literally. Thus, the formal operators may well have rather immediate neurophysiological correlates (Hanazawa \& Komatsu, 2001).

The formal theory is most easily developed for the case of Gaussian random fields, since for such surfaces the required statistics are easily obtained. In practice, though, we have found the formal theory to apply remarkably well to a wide range of natural surfaces.

Developing the theory for anisotropic Gaussian surfaces is conceptually straightforward, if extremely tedious in practice (the calculation is best done with a symbolic math package on the computer). We present the gist of the derivation in the Appendix. By doing this calculation, one derives closed-form analytic expressions, though these formulas are so complicated as to be intuitively impenetrable. Thus, one has to study either numerical cases or low-order approximations. Either method turns out to be quite useful.

In Figure 4 we explain our angular coordinates, and in Figure 6 we show some numerical predictions for the case of the squared gradient. In the latter figure, the deviations 
A

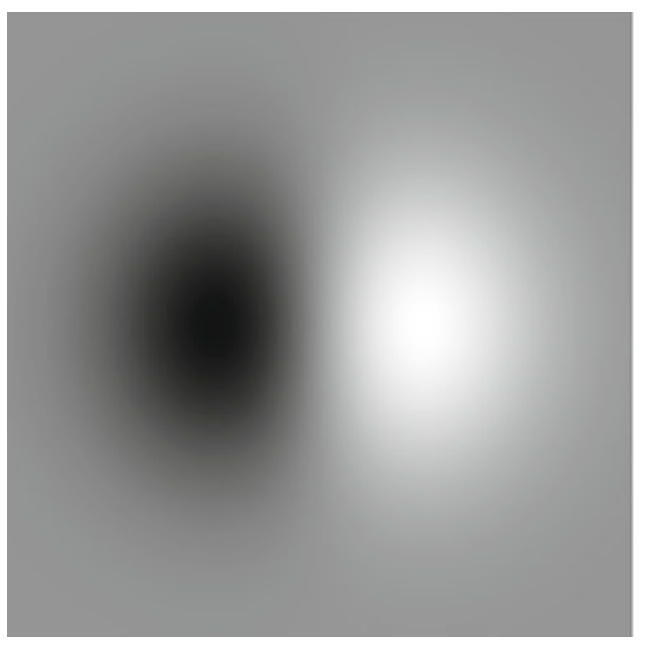

B

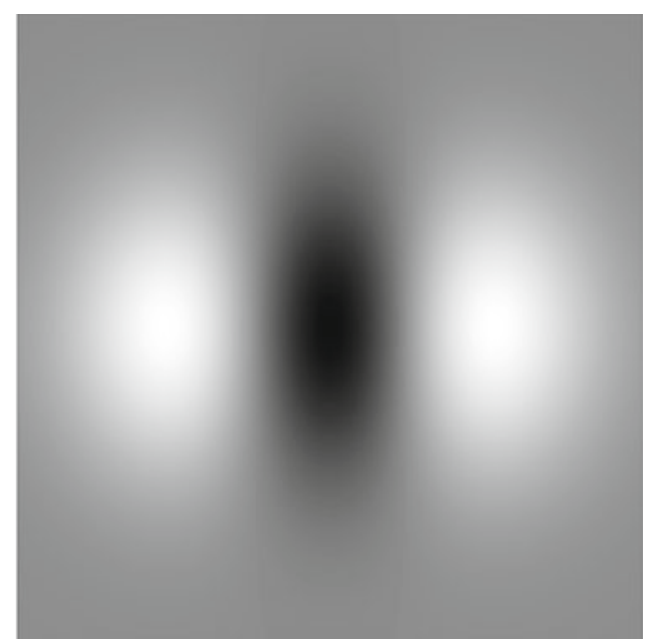

Figure 5. (A) An "edge detector," or image operator that finds the first-order directional derivative of the image intensity at a certain scale. (B) A "line detector," or image operator that finds the second-order directional derivative of the image intensity at a certain scale. Such operators can of course be constructed for any direction (here, for horizontal). Notice the similarity of these basic mathematical operators to the simple cells of the visual cortex.

from veridicality (in degrees) of the estimated surface illuminance flow have been plotted as a function of the angle subtended by the direction perpendicular to the axis of elongation and the direction of surface illuminance flow. The curves are for different degrees of anisotropy.

As it turns out, a sharp transition of behavior exists at a certain critical value of the anisotropy. (This is easily discovered in numerical simulations, but can also be shown analytically, though this involves rather complicated formalisms.) The critical case is when the aspect ratio of the autocorrelation function equals $\sqrt{3}=1.73 \ldots$ For the moderate-anisotropy regime, the systematic errors can run as high as $45^{\circ}$ (with a limiting case shown by the broken curve), though for low anisotropy the values will be much smaller. Although (very) significant systematic errors occur, the predictions are determined largely by the flow direction. For the high-anisotropy regime, we see a type of behavior for which it is essentially impossible to derive the orientation of surface illuminance flow. In this case, the estimates are largely determined through the orientation of the axis of elongation rather than through the flow direction.

In the low-anisotropy regime, we have the following equations to the first order in the anisotropy parameter $\Xi$ (i.e., the aspect ratio of the autocorrelation function of the roughness minus 1 ; thus, $\Xi=0$ is the isotropic case, $\Xi \approx 1$ is markedly anisotropic, and so forth):

$$
\begin{aligned}
& \Delta \varphi_{\mathrm{g}}=(3 / 2) \Xi \sin 2 \varphi+O\left(\Xi^{2}\right), \\
& \Delta \varphi_{\mathrm{H}}=2 \Xi \sin 2 \varphi+O\left(\Xi^{2}\right),
\end{aligned}
$$

where $\Delta \varphi_{\mathrm{g}, \mathrm{H}}$ denote the systematic deviation for the gradient and the Hessian, respectively, and $\varphi$ is the direction of surface illuminance flow relative to the axis of elongation of the surface troughs and ridges (the angle $\varphi$ is defined in Figure 4). These formulas should yield useful predictions for the systematic errors committed by human observers in the case of moderate anisotropic roughness.

\section{The Experiment}

Three observers (the authors) participated in the experiment. All 3 have ample experience with illuminance flow estimation for the case of isotropic random surfaces. We investigated one case in the high-anisotropy regime, simply to verify that this task is very difficult and that judgments line up with the direction orthogonal to the axis of elongation of the roughness rather than with the illuminance flow direction. We devoted a larger effort to the moderate-anisotropy regime, in which a pattern of systematic errors is to be expected. We selected an anisotropy of $\Xi=0.2$, which is clearly in the moderate-anisotropy regime, but large enough to cause large (theoretical) deviations, maximally $17.19^{\circ}$ for the gradient and $22.19^{\circ}$ for the Hessian model.

\section{METHOD}

We used the same methods as in our studies on isotropic roughness (Koenderink et al., 2003). In summary, stimuli were computer generated, taking both shading and cast shadows into account but ignoring vignetting and multiple scattering. This should be a good approximation for nottoo-high relief. Independent statistical samples of Gaussian noise were used for each image. We prepared stimuli for many orientations of the direction of illuminance flow relative to the axis of elongation. Both the direction of illuminance flow and the orientation of the axis of elongation were randomized, viewing of the surfaces was from the normal direction, and the elevation of the source was kept constant (in the true-shading regime).

For the case of the high-anisotropy regime, each observer performed one session on a set of 250 samples. For 


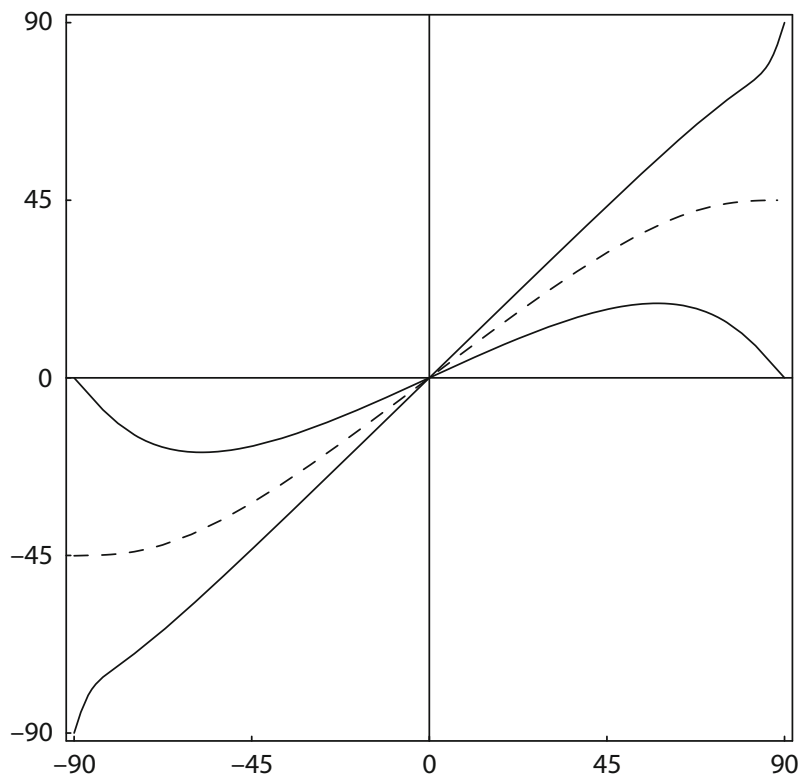

Figure 6. Theoretical curves showing the error in judgments on the basis of the gradient squared structure tensor for various values of the anisotropy. On the abscissa is the angle $\varphi$, which is subtended by the illuminance flow direction and the direction perpendicular to the axis of elongation. The broken curve represents a critical condition: For relatively small anisotropies, the graph undulates about the horizontal; for relatively high anisotropies, the graph undulates about the diagonal. In the former case, the predicted values are dominated by the actual direction of illuminance flow, whereas in the latter case, they are dominated by the axis of elongation.

the case of the moderate-anisotropy regime, each observer did five sessions on a single set of 250 samples. This design generated amply sufficient data to check the validity of the theoretical predictions.

\section{RESULTS}

As expected, in the case of the high-anisotropy regime $(\Xi=1)$, the observers often failed to have the impression of an illuminated surface. They found it difficult to perform the task properly in this condition, but were nevertheless required to. The results of those sessions are shown in Figure 7. The systematic deviations are very high, and in fact the judgments are dominated by the axis of elongation more than by the true orientation of illuminance flow. The theoretical prediction for the assumption of squared gradient statistics (dotted curve) turns out to describe the data quite well.

In the case of moderate to small anisotropy $(\Xi=0.2)$, the results are much closer to veridicality (Figure 8). Nevertheless, there remain very significant systematic deviations from veridicality. Since the deviations in this condition were not very large (always less than $20^{\circ}$ ), we repeated the session (on the same 250 samples) five times in order to improve the reliability of the data. The error bars shown in the graph are the standard errors computed over the average results of the sessions. Evidently, the systematic deviations are very significant and follow a definite pattern.
We compared the results with the theoretical prediction based on the squared gradient statistics, which is $\Delta \varphi_{\mathrm{g}}=17.19^{\circ} \sin 2 \varphi$. The fit is reasonable for all observers, whereas the fit with the prediction based on the squared Hessian statistics is numerically much inferior. (For the Hessian statistics, the predicted deviations are $33 \%$ higher.) By pooling all observers (which appears reasonable, given the very similar results), automatically omitting two outliers per observer, and computing the $\chi^{2}$ merit function, we obtained 39.685 for the gradient model and 111.842 for the Hessian model, with $21 d f$. Thus, we obtained $Q=.016$ for the gradient and $Q=4.4 \times 10^{-14}$ for the Hessian model (two-sided $p$ values). We conclude that we may accept the gradient model as accounting for the data, whereas we have to reject the Hessian model.

\section{CONCLUSIONS}

The results from the experiment are clearly in good agreement with the initial hypothesis, which was that the observers might simply (mis)apply an algorithm fit for the analysis of the illuminance texture of an illuminated isotropic rough surface to instances of illuminated anisotropic rough surfaces.

This result is surprising in a way, because it is certainly possible to account for the anisotropy in the moderateanisotropy regime. Because the predictions of the algorithms (for an anisotropic surface) based on squared gradient and squared Hessian statistics are different, both can be combined to solve for the anisotropy and arrive at a corrected, veridical orientation of surface illuminance flow. Such a scheme is easy enough to implement as a machine vision algorithm, and it performs as expected. Thus, the visual cues are certainly there, and the fact that human observers fail to account for the effect of moderate

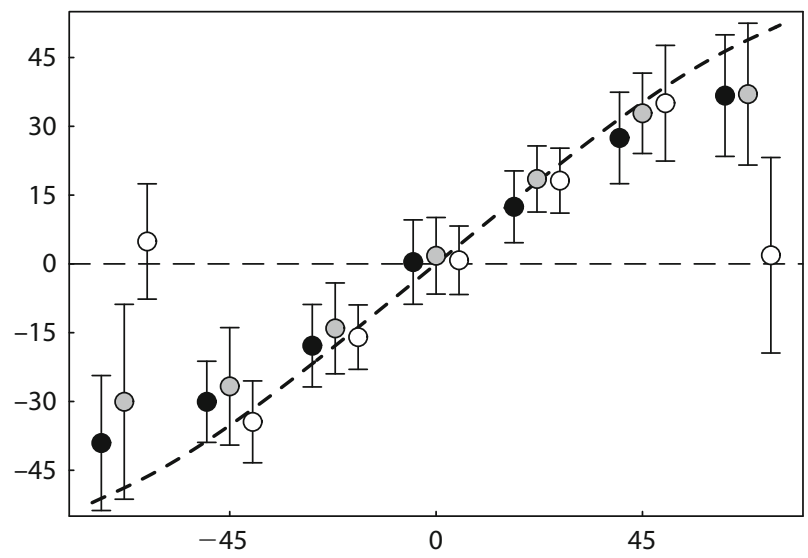

Figure 7. The systematic errors in the high-anisotropy regime $(\Xi=1)$ for all observers as a function of the angle $\varphi-$ that is, the angle subtended by the true illuminance direction and the direction perpendicular to the axis of elongation. The curve is the theoretical prediction for the case of the gradient squared statistics. The black data points are for observer A.J.v.D., gray for J.J.K., and white for S.C.P. (The data points for observers A.J.v.D. and S.C.P. have been displaced slightly toward the left and right, respectively, in order to avoid unfortunate superpositions.) 


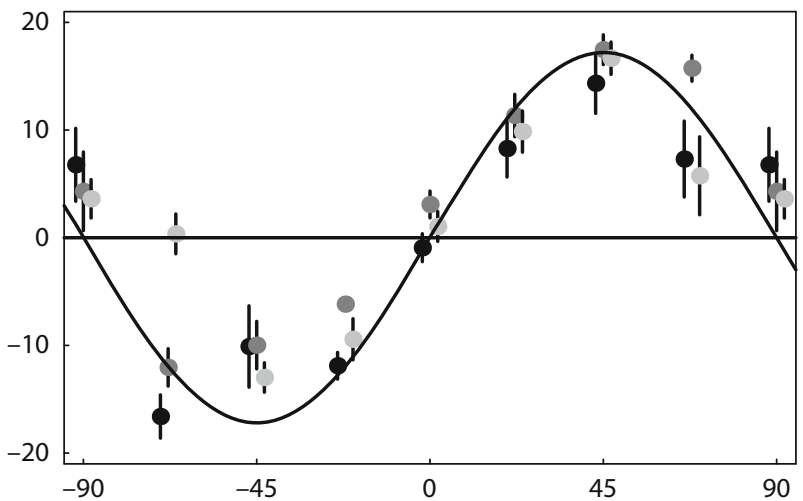

Figure 8. The systematic errors in the moderate-anisotropy regime $(\Xi=0.2)$ for all observers as a function of the angle $\varphi$ - that is, the angle subtended by the true illuminance direction and the direction perpendicular to the axis of elongation. The curve is the theoretical prediction for the case of the gradient squared statistics. The black data points are for observer A.J.v.D., dark gray for J.J.K., and light gray for S.C.P. (The data points for observers A.J.v.D. and S.C.P. have been displaced slightly toward the left and right, respectively, in order to avoid unfortunate superpositions.) Notice that the vertical scale has been expanded relative to that in Figure 7 . The vertical bars denote the standard errors across repeated settings.

anisotropy reveals a specific kind of "cue blindness." We must assume that both the squared gradient and squared Hessian statistics are available to the brain, because they exist in the activities of pools of edge detectors and line detectors, both known to be present in the primary visual cortex (Hubel, 1988). Despite the fact that the information is - in principle - available in V1, the human visual system apparently ignores it. Perhaps it is not worth the trouble to implement such an ability; we do not have sufficient understanding of the relevant ecological data to be able to decide the issue. Another possibility is that the gradient and Hessian statistics are simply pooled, and the gradient statistics then apparently dominate. This possibility seems virtually impossible to check, since the relevant neurophysiological parameters are unknown.

It is not immediately evident whether these conclusions will carry over to natural materials. In natural surfaces, one typically finds a spectrum of scales (since the surfaces involved often have a fractal character), whereas the Gaussian random surfaces used in our experiments are characterized by a single scale. This issue certainly deserves closer attention. Another point concerns the fact that we only looked at planar, frontoparallel surfaces, whereas objects in the daily environment are generically curved, thus offering a variety of surface attitudes simultaneously and significantly increasing the possibilities for successful inferences. A significant theoretical development would be required in order to address these problems fully.

We find that the squared gradient statistics fit the present data significantly better than the squared Hessian statistics do. This result perhaps suggests that illuminance flow detection in human vision is based on the activity of edge detectors (not line detectors) in the primary visual cortex. It would be of interest to attempt to devise empirical methods to decide this issue more definitively.

\section{AUTHOR NOTE}

This work was sponsored via the European program VISIONTRAIN, contract No. MRTN-CT-2004-005439. S.C.P. was supported by the Netherlands Organization for Scientific Research (NWO). Correspondence relating to this article may be sent to J. J. Koenderink, Physics and Astronomy, Buys Ballot Laboratory, Princetonplein 5, 3584CC Utrecht, The Netherlands (e-mail: j.j.koenderink@phys.uu.nl).

\section{REFERENCES}

Belhumeur, P. N., Kriegman, D., \& Yuille, A. (1999). The bas-relief ambiguity. International Journal of Computer Vision, 35, 33-44.

FÖRSTNER, W. (1994). A framework for low level feature detection. In J.-O. Eklundh (Ed.), Computer vision, ECCV'94 (Vol. 2, pp. 383394). New York: Springer.

Gershun, A. (1939). The light field (P. Moon \& G. Timoshenko, Trans.). Journal of Mathematics \& Physics, 18, 51-151.

Hanazawa, A., \& Komatsu, H. (2001). Influence of the direction of elemental luminance gradients on the responses of V4 cells to textured surfaces. Journal of Neuroscience, 21, 4490-4497.

Harris, C. G., \& Stevens, M. J. (1988, August). A combined corner and edge detector. Paper presented at the 4th Alvey Vision Conference, Manchester, U.K.

Horn, B. K. P., \& Brooks, M. J. (EDs.) (1989). Shape from shading. Cambridge, MA: MIT Press.

Hubel, D. H. (1988). Eye, brain, and vision. New York: Freeman.

Knutsson, H. (1989). Representing local structure using tensors. In M. Pietikainen \& J. Rohning (Eds.), Proceedings of the 6th Scandinavian Conference on Image Analysis (pp. 244-251). Oulu, Finland: Pattern Recognition Society of Finland.

KoenderinK, J. J., \& PONT, S. C. (2002). Texture at the terminator. In 1st International Symposium on 3D Data Processing Visualization and Transmission (3DPVT 2002) (pp. 406-415). New York: IEEE Press.

Koenderink, J. J., van Doorn, A. J., Kappers, A. M. L., te Pas, S. F., \& PonT, S. C. (2003). Illumination direction from texture shading. Journal of the Optical Society of America A, 20, 987-995.

Koenderink, J. J., van Doorn, A. J., \& Pont, S. C. (2004). Light direction from shad(ow)ed random Gaussian surfaces. Perception, 33, 1405-1420.

KöTHE, U. (2003). Edge and junction detection with an improved structure tensor. In B. Michaelis \& G. Krell (Eds.), Pattern recognition: Proceedings of the 25th DAGM Symposium, Magdeburg 2003 (pp. 25-32). Heidelberg: Springer.

Lambert, J. H. (1760). Photometria, sive, De mensura et gradibus luminis, colorum et umbrae. Augsburg: E. Klett.

Moon, P., \& Spencer, D. E. (1981). The photic field. Cambridge, MA: MIT Press.

Penirschke, A., Chantler, M. J., \& Petrou, M. (2002). Illuminant rotation invariant classification of 3D surface textures using Lissajous's ellipses. In M. J. Chantler (Ed.), Proceedings of Texture 2002, the 2nd International Workshop on Texture Analysis and Synthesis (pp. 103-107). Glasgow: Heriot-Watt University.

Pentland, A. P. (1982). Finding the illuminant direction. Journal of the Optical Society of America, 72, 448-455.

Pont, S. C., \& KoEnderinK, J. J. (2003). Illuminance flow. In Computer Analysis of Images and Patterns, 10th International Conference, CAIP 2003, Groningen, The Netherlands, August 25-27, 2003 (pp. 90-97). Heidelberg: Springer.

Pont, S. C., \& KoEnderinK, J. J. (2004). Surface illuminance flow. In 2nd International Symposium on 3D Data Processing, Visualization and Transmission (3DPVT 2004) (pp. 2-9). New York: IEEE Press.

RÄTH, C., \& MoRfILL, G. (1997). Texture detection and texture discrimination with anisotropic scaling indices. Journal of the Optical Society of America A, 14, 3208-3215.

Todd, J. T., Oomes, A. H. J., KoenderinK, J. J., \& Kappers, A. M. L. 
(2002). The perception of 3D shape from anisotropic texture patterns [Abstract]. Journal of Vision, 2(7), 113a.

van DigGelen, J. (1959). Photometric properties of lunar crater floors. Recherches Astronomiques de l'Observatoire d'Utrecht, 14, 1-114.
VARMA, M., \& Zisserman, A. (2004). Estimating illumination direction from textured images. In Proceedings of the 2004 IEEE Computer Society Conference on Computer Vision and Pattern Recognition (CVPR04) (pp. 1063-1069). New York: IEEE Press.

\section{APPENDIX}

\section{Formal Structure of Illuminance Flow}

The physical basis of classical shape from shading is simply Lambert's surface attitude effect, which dates from the 18th century. If $n$ denotes the outward surface normal, and $i$ the direction toward the light source (both assumed to be unit vectors), the surface irradiance caused by the incident beam is proportional to the inner product $\boldsymbol{n} \cdot \boldsymbol{i}$. For a Lambertian surface, the radiance of the scattered beam in arbitrary directions is proportional with the irradiance, and thus, so is the "image intensity" (this is the term commonly used in computer vision and graphics - perhaps unfortunately, because it is not a standard radiometric term). Thus, the image intensity at any point is proportional with the cosine of the obliquity of the incident beam at the corresponding surface location. More specifically, for a surface albedo $\rho(u, v)$ (where $\{u, v\}$ denote parameters on the surface) and an incident beam of radiance $N$, one has

$$
I(u, v)=(1 / \pi) \rho(u, v) N \cdot[\boldsymbol{n}(u, v) \cdot \boldsymbol{i}],
$$

where $I(u, v)$ denotes the image intensity at the location in the image corresponding to the surface location $\{u, v\}$. Conventionally, one assumes $\rho(u, v)=\rho_{0}$, a constant (and often, even $\rho_{0}=1$ ), and parameterizes the surface via the (Cartesian) image coordinates $\{x, y\}$ (with $z$ denoting the depth coordinate). In the case of orthographic projection, one then has

$$
I(x, y)=(1 / \pi) \rho_{0} N \cdot[\boldsymbol{n}(x, y) \cdot \boldsymbol{i}]=(1 / \pi) \rho_{0} N \cdot\left[i_{z}-\left(i_{x} z_{x}+i_{y} z_{y}\right)\right] / \sqrt{ }\left(1+z_{x}^{2}+z_{y}^{2}\right)
$$

and strives to solve for $z(x, y)$ (the "surface relief") and the direction of illumination $i$. (Here we use the notation $z_{x}$ for $\partial z / \partial x$, and so forth, for the sake of clarity.)

In this setting, it is evidently only the normal component $\boldsymbol{i}_{\perp}=(\boldsymbol{n} \cdot \boldsymbol{i}) \boldsymbol{n}$ of the direction of the incident beam with respect to the surface that is (at least partially) observable through the shading.

In the lowest-order approximation that conserves contrast variation, the contrast in the neighborhood of a point $x=y=0$ at which $z_{x}=z_{y}=0$ is

$$
C(x, y)=-\left(i_{x} / i_{z}\right) z_{x}-\left(i_{y} / i_{z}\right) z_{y}+\ldots
$$

Use of this contrast is convenient, because the constant factor $\rho_{0} N / \pi$ cancels out and only the visually relevant variation is retained. This is the "low relief" approximation - that is, $z_{x}^{2}+z_{y}^{2}<<1$.

Cursory examination of actual photographs reveals that most surfaces are rough on the microscale; that is, fluctuations of the surface normal that are finer than some fiducial scale are considered to be due to "roughness" rather than "shape" (i.e., surface relief). Such roughness has been observed to lead to an image "texture" that depends mainly on the tangential component $\boldsymbol{i}_{/ /}=\boldsymbol{i}-\boldsymbol{i}_{\perp}$ of the direction of incidence. For low relief, the root mean square contrast is proportional with $\cot \theta$, where $\theta=\arctan \left[\sqrt{ }\left(i_{x}^{2}+i_{y}^{2}\right) / i_{z}\right]$ is the elevation of the illumination. The contrast explodes near the terminator of the attached shadow, an effect that can frequently be observed in natural scenes and is well-known to visual artists.

However, the mere magnitude of the contrast of illuminance texture is not the only relevant factor; the contrast also has an important statistical structure, for it can be shown that for a Gaussian random modulation on the frontoparallel plane, the direction of the largest eigenvalue of either the structure tensor $\langle\boldsymbol{g} \cdot \boldsymbol{g} \dagger\rangle$ or $\langle\boldsymbol{H} \cdot \boldsymbol{H} \dagger\rangle$ [where $\boldsymbol{g}$ denotes the contrast gradient $\nabla C(x, y)$ and $\boldsymbol{H}$ the contrast Hessian $\nabla \nabla C(x, y)$ ] lies in the plane of incidence. Here, the operator $\langle\ldots\rangle$ denotes a local spatial average at a scale coarser than the microscale - that is, at a scale at which the surface appears smooth. The direction of the local plane of incidence thus has to be considered an additional "observable."

For the spatial average of the squared gradient, one finds

$$
<\boldsymbol{g} \cdot \boldsymbol{g}^{\dagger>}=\left(\begin{array}{c}
a_{11} a_{12} \\
a_{21} a_{22}
\end{array}\right),
$$

with

$$
\begin{aligned}
& a_{11}=<z_{x x}^{2}>i_{x}^{2}+2<z_{x x} z_{x y}>i_{x} i_{y}+<z_{x y}^{2}>i_{y}^{2}, \\
& a_{12}=<z_{x y} z_{x x}>i_{x}^{2}+<z_{x y} z_{y y}>i_{y}^{2}+\left(<z_{x x} z_{x y}>+<z_{x y}^{2}>\right) i_{x} i_{y}, \\
& a_{21}=a_{12}
\end{aligned}
$$

and

$$
a_{22}=<z_{x y}^{2}>i_{x}^{2}+2<z_{x y} z_{y y}>i_{x} i_{y}+<z_{y y}^{2}>i_{y}^{2}
$$




\section{APPENDIX (Continued)}

Notice that the matrix depends both on the direction of illumination $i$ and the statistical structure of the surface, as specified by the averages of products of second-order spatial derivatives $\left\langle z_{i j} z_{k l}\right\rangle$, where $i, j, k, l=x$ or $y$. For the squared Hessian, one obtains a similar (though much longer) expression that depends on the averages of products of third-order spatial derivatives $\left\langle z_{i j k} z_{l m n}\right\rangle$, where $i, j, k, l, m, n=x$ or $y$. The average products of partial derivatives can be evaluated exactly in few cases, but one of these is the comparatively simple case of a random Gaussian surface. For instance, for a Gaussian surface with a spatial power spectrum

$$
S\left(k_{x}, k_{y}\right)=\left(2 \pi \sigma^{2} / \xi\right) \exp \left\{-\sigma^{2}\left[\left(k_{x} \sin \psi-k_{y} \cos \psi\right)^{2}+\xi^{2}\left(k_{x} \cos \psi+k_{y} \sin \psi\right)^{2}\right] /\left(2 \xi^{2}\right)\right\}
$$

(notice here that the autocorrelation function and the power spectrum are Fourier transform pairs), where $\boldsymbol{k}$ is the wave vector, $\sigma^{2}$ the variance, $\xi$ a measure of anisotropy (with $\xi=1$ as the isotropic case), and $\psi$ the orientation of the anisotropy, one would generate the equations

$$
\begin{aligned}
& <z_{x x}^{2}>=3 \pi^{2}\left[1+\xi^{2}-\left(\xi^{2}-1\right) \cos 2 \psi\right]^{2} / \sigma^{4}, \\
& <z_{x x} z_{x y}>=3 \pi^{2}\left[2\left(1-\xi^{4}\right) \sin 2 \psi+\left(\xi^{2}-1\right)^{2} \sin 4 \psi\right] /\left(2 \sigma^{4}\right), \\
& <z_{x y}^{2}>=\pi^{2}\left[3+2 \xi^{2}+3 \xi^{4}-3\left(\xi^{2}-1\right)^{2} \cos 4 \psi\right] /\left(2 \sigma^{4}\right), \\
& <z_{x x} z_{y y}>=<z_{x y}^{2}>, \\
& <z_{x y} z_{y y}>=\left\{-3 \pi^{2}\left(\xi^{2}-1\right)\left[1+\xi^{2}+\left(\xi^{2}-1\right) \cos 2 \psi\right] \sin 2 \psi\right\} / \sigma^{4},
\end{aligned}
$$

and

$$
<z_{y y}^{2}>=3 \pi^{2}\left[1+\xi^{2}+\left(\xi^{2}-1\right) \cos 2 \psi\right]^{2} / \sigma^{4} .
$$

The results for the averages of the products of third-order partial derivatives would yield similar — though much longer-expressions.

Using these results, it is in principle straightforward to find the eigenvalues and the eigenvectors for these matrices. For the isotropic case, it turns out that the results differ only by a factor. For the anisotropic case, the results are different (as expected), and the resulting expressions are very involved. A clearer impression of the nature of the result is obtained through a Taylor expansion in terms of powers of $(\xi-1)$. Up to the second order, these results are

$$
\begin{aligned}
& \varphi_{\mathrm{g}}=\varphi+(3 / 2)(\xi-1) \sin 2(\varphi-\psi)-(3 / 4)(\xi-1)^{2}[\sin 2(\varphi-\psi)-2 \sin 4(\varphi-\psi)]+\ldots, \\
& \varphi_{\mathrm{H}}=\varphi+2(\xi-1) \sin 2(\varphi-\psi)-(\xi-1)^{2}[\sin 2(\varphi-\psi)-(11 / 4) \sin 4(\varphi-\psi)]+\ldots
\end{aligned}
$$

These are the expressions used in the article. 